\title{
Dilute polymer solution drops impacting on heated surfaces: New impact morphologies and impact regime maps
}

\author{
Chetan Lakhanpal ${ }^{1}$, Volfango Bertola*1 \\ ${ }^{1}$ School of Engineering, University of Liverpool, United Kingdom \\ ${ }^{*}$ Corresponding author: Volfango.Bertola@liverpool.ac.uk
}

\begin{abstract}
The impact morphology of dilute polymer solution drops on a heated surface is studied experimentally by means of high-speed imaging, with respect to the following parameters: surface temperature; impact Weber number; polymer concentration; polymer molecular weight. In addition to impact morphologies observed in Newtonian drops (deposition, rebound, secondary atomisation and breakup/splashing), three new impact regimes have been identified: (i) a single satellite droplet ejected in the direction of bouncing but tethered to the main drop by a thin liquid filament; (ii) a splashing-like behaviour (semi-splashing), where the rim instability generates satellite droplets tethered to the lamella by thin liquid filaments; (iii) a spray-like behaviour (semi-spray), where a fine secondary atomisation generated upon impact is quickly absorbed back into the drop globule. Experiments were carried out using drops of aqueous polyethylene oxide (PEO) solutions, with mass concentrations of $100 \mathrm{ppm}$, $200 \mathrm{ppm}$ and $400 \mathrm{ppm}$, and PEO molecular weights of $2 \mathrm{MDa}, 4 \mathrm{MDa}$, and $8 \mathrm{MDa}$. The impact morphology on a polished aluminium surface with temperatures ranging between $160^{\circ} \mathrm{C}$ and $400^{\circ} \mathrm{C}$ was investigated for impact Weber numbers between 20 and 170, taking side view images of impacting drops at a rate of 1,000 frames per second.
\end{abstract}

\section{Keywords}

Drop Impact; Heated Surfaces; Polymer Solutions; Impact Regime Map.

\section{Introduction}

The dynamics of liquid drops impinging on a heated surface is a phenomenon of interest in many areas of engineering, including spray cooling, inkjet printing for advanced manufacturing applications, quenching of alloys in the steel industry and nuclear reactor safety. Despite several decades of research, this phenomenon is understood only to a limited extent and requires further research; specifically, the development of impact regime maps (IRMs) is of practical interest because they provide a consolidated understanding of the impact behaviour and represent a tool of practical use in the design of nozzles, print heads, etc. A quantitative and comprehensive IRM also presents an important test case for the development and validation of theoretical models describing specific features, such as transitions between different impact regimes.

Currently, an extensive body of literature on the behaviour of Newtonian drops impacting on heated surfaces exists. The first IRM produced [1] identified most of the impact regimes, however lacked clear quantitative description on the initiation and extent of these regimes. This work was soon followed by a detailed investigation that allowed production of three distinct IRMs corresponding to Weber numbers of 20, 60 and 220 and for surface temperatures ranging from $100^{\circ}$ to $300^{\circ} \mathrm{C}$ [2]. Although this work included substantial information on the spreading behaviour of a drop and its heat transfer characteristics, the IRMs were far from being fully comprehensive, particularly lacking information on all possible impact regimes that can be realised as well as the transitional boundaries. This was partly because of the limitations in photographic techniques available for analysis at the time. Moreover, the photographic results reported were not based on studying the lifetime of a single drop but rather based upon an ensemble principle of several drops, with one photograph taken per drop. Other semi-qualitative maps were proposed in the following years $[3,4]$

Drop impact was investigated for liquid drops impacting upon a nickel surface with angle of incidence ranging between $10^{\circ}$ and $80^{\circ}$ [5]. The impact regime map developed as a result uses as coordinates the dimensionless temperature, $\mathrm{T}^{*}$, defined as

$$
T^{*}=\frac{T_{\mathrm{w}}-T_{\mathrm{b}}}{T_{1}-T_{\mathrm{b}}}
$$

and the K-number $[6,7]$ 


$$
\mathrm{K}=\mathrm{We} \mathrm{Oh}^{-0.4}
$$

where $T_{w}-T_{b}$ is the wall superheat temperature, i.e. the difference between wall temperature and the boiling point of the fluid, $T_{1}$ is the Leidenfrost temperature .

For cases where the wall temperature is lower than the Leidenfrost temperature, the proposal was to replace $T_{w}$ in equation (2) by effective contact temperature as described in the expression (3) below [8].

$$
T_{w}=\frac{\varepsilon_{\mathrm{w}} T_{\mathrm{TH}}+\varepsilon_{1} T_{1}}{\varepsilon_{w}+\varepsilon_{1}}
$$

where $T_{H}$ and $T_{1}$ denote the wall and liquid temperatures respectively and $\varepsilon_{w}$ and $\varepsilon_{1}$ are the respective thermal effusivities of the wall and the liquid. This map identifies three impact regimes: deposition, splashing and rebound and displays qualitative transition boundaries; the Leidenfrost temperature is assumed to be $220^{\circ} \mathrm{C}$, however this quantity depends on the Weber number and, for water drops interacting with polished aluminium surfaces, has a minimum value of approximately $160^{\circ} \mathrm{C}$ when We $\rightarrow 0$ [9-11]. A comprehensive review on single droplet impact research and its relevance to understanding fuel sprays impingement can be found in [12].

To rationalise the rich variety of impact morphologies observed for Newtonian drops impinging on heated surfaces, it was proposed to identify "simple" impact regimes, displaying one distinctive feature (deposition, rebound, splashing/breakup) and "mixed" regimes, resulting from the combination of simple regimes with secondary atomisation [13]. Such unifying classification on one hand embraces the different impact morphologies reported in the existing literature, and on the other hand is simple enough to be used for practical purposes; in addition, it allows one to derive simple models for transition boundaries.

Results about Newtonian drop impact on heated surfaces reported in the literature are generally consistent with one another, to the exception of one recent work presenting an IRM based upon the impact of water and FC-72 drops upon silicon and sapphire surfaces, for temperatures between $200^{\circ} \mathrm{C}$ and $600^{\circ} \mathrm{C}$ and the Weber numbers between 0.5 and 600 [14]. Whilst one may argue the differences between these results and the rest of the literature are due to the high smoothness of the surfaces used, a more careful analysis suggests they may be caused by the design of the experimental setup, which leads to overestimate the surface temperature [13].

The impact of non-Newtonian drops on heated surfaces received comparatively less attention. Early studies focused primarily on dilute polymer solution drops, finding significant differences with respect to Newtonian drops [15]; in particular, it was observed that adding small amounts of a flexible polymer to the aqueous phase, secondary atomisation can be suppressed completely [16], and droplet rebound in the Leidenfrost regime is significantly enhanced [17]. More recently, it was shown that Leidenfrost rebounds are only weakly affected by the polymer concentration (i.e., by the fluid rheology) [18], but can be related to the symmetry of the rebound process [19]. Finally, the study of non-Newtonian drop impact in the Leidenfrost regime was extended to viscoplastic gels [20], showing that the fluid yield stress can suppress drop rebound.

The aim of the present work is to provide a systematic analysis of the impact morphology of dilute polymer solution drops, to produce impact regime maps for different polymer molecular weights and concentrations.

\section{Material and methods}

Test fluids were prepared by dissolving polyethylene oxide (PEO) in de-ionized water with mass concentrations of 100,200 , and $400 \mathrm{ppm}$; the polymer had average molecular weights of $2 \mathrm{MDa}, 4 \mathrm{MDa}$, and $8 \mathrm{MDa}$, and typical density of $1210 \mathrm{~kg} / \mathrm{m}^{3}$. In total, five distinct batches were produced:

- $\quad \mathrm{S} 1$ : Molecular weight $=4 \mathrm{MDa}$; Concentration $=100 \mathrm{ppm}$

- S2: Molecular weight $=4 \mathrm{MDa}$; Concentration $=200 \mathrm{ppm}$

- S3: Molecular weight $=4 \mathrm{MDa}$; Concentration $=400 \mathrm{ppm}$

- S4: Molecular weight $=2 \mathrm{MDa}$; Concentration $=200 \mathrm{ppm}$

- S5: Molecular weight $=8 \mathrm{MDa}$; Concentration $=200 \mathrm{ppm}$

In all cases, the polymer concentration was smaller than the overlap concentration, which can be estimated through the Mark-Houwink equation [18], therefore all test fluids are dilute solutions. The test fluid viscosities ranged between $1.1 \mathrm{mPa} \mathrm{s}$ and $1.5 \mathrm{mPa} \mathrm{s}$, while their surface tension was almost the same as that of the pure solvent $(\sim 70 \mathrm{mN} / \mathrm{m})$ [18].

Drops of test fluids were released from a blunt hypodermic needle (gauge 21, i.d. $0.495 \mathrm{~mm}$ ) and impacted upon the polished top surface of a square aluminium block $(40 \mathrm{~mm} \times 40 \mathrm{~mm})$ containing two $100 \mathrm{~W}$ cartridge heaters. The surface temperature was controlled by a PID controller driven by a thermocouple inserted into the aluminium block, approximately $1 \mathrm{~mm}$ below the impact point, and was varied in increments of $20^{\circ} \mathrm{C}$ between $160^{\circ} \mathrm{C}$ and $400^{\circ} \mathrm{C}$, with an accuracy of $\pm 1^{\circ} \mathrm{C}$. A schematic of the experimental setup is displayed in Figure 1. 
The drop diameter at equilibrium was calculated from the drop mass, $m$, measured with a precision scale (Mettler Toledo) as $D_{0}=\sqrt[3]{6 m / \pi \rho}$, where $\rho$ is the fluid density. Averages over 50 samples returned a value of $D_{0}=2.93 \pm$ $0.1 \mathrm{~mm}$.

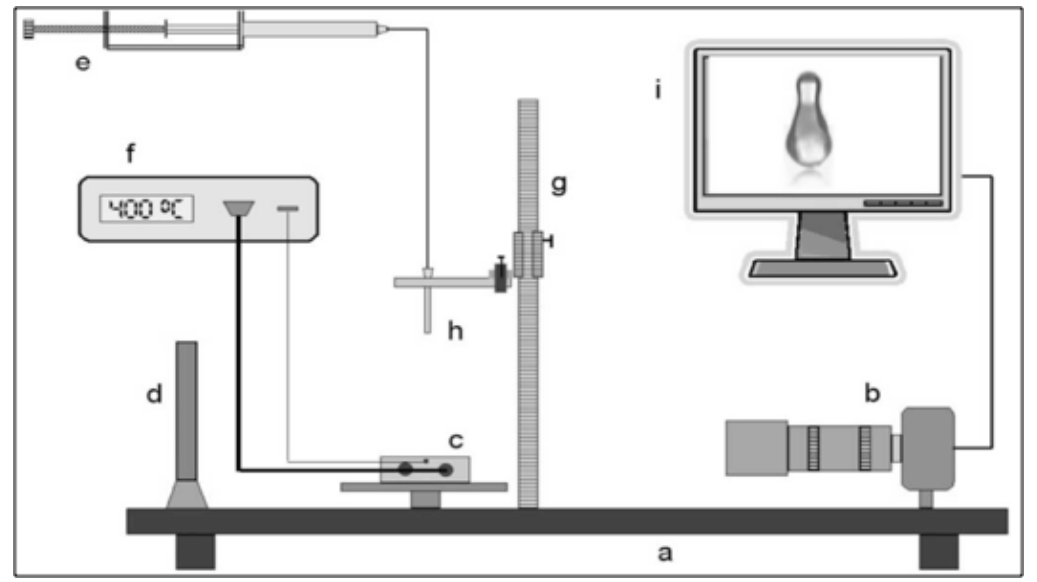

Figure 1. Schematic of experimental setup: a) optical breadboard; b) high-speed camera; c) heated aluminium block; d) LED backlight; e) drop dispensing system; f) temperature controller; g) digital vernier callipers; h) needle; i) computer.

The drop falling height, $\mathrm{H}$, was adjusted using a digital height gauge, and the free-fall impact velocity, $\mathrm{u}$, was calculated as $\sqrt{2 g\left(H-D_{0}\right)}$; previous experiments showed this is almost identical to the measured impact velocity, for falling heights up to $30 \mathrm{~cm}$ [17]. In the present study, the drop falling height was varied within the range from $30 \mathrm{~mm}$ to $200 \mathrm{~mm}$, corresponding to Weber numbers, between 23 and 166 .

The impact of single drops was recorded using a high-speed CMOS camera (Mikrotron MC1310) at the rate of 1000 frames per second, with back-to-front illumination. For each surface temperature and Weber number, the experiment was repeated 10 times for the sake of statistical coherence.

The recorded videos were processed in Labview environment. The processed frames obtained as a result were used to produce pictorial maps for each of the five specified combinations. These pictorial maps use surface temperature and the Weber number as the coordinates and apart from showing the impact behaviour, also allow one to make a qualitative assessment of the boundaries between the various impact regimes. The data was also processed in Matlab environment to generate impact regime maps, showing the impact behaviour as a function of surface temperature and Weber number. More details about the experimental apparatus and procedure can be found in previous works $[13,17,18]$.

\section{Results and discussion}

The impact regimes observed for water drops impacting on a heated surface, based on the simplified classification proposed in [13], i.e., secondary atomisation (SA), rebound, (R), breakup (B) and/or splashing (S), rebound with secondary atomisation (RSA), and breakup with secondary atomisation (BSA), are also observed in case of dilute polymer solution drops, and will not be discussed further in the present work. Besides these conventional impact regimes, dilute polymer solution drops may exhibit other impact morphologies, depending on the Weber number, the impact surface temperature, the polymer concentration and molecular weight.

At low polymer concentrations, there is a range of Weber numbers where a single satellite drop separates in the vertical direction during rebound, shortly after the drop has bounced off the surface; this drop is tethered to the main drop body by a thin liquid filament, which is subject to uniaxial stretching, and does not break up until the two droplets re-coalesce into a single drop, as shown in Figure 2. The diameter of the single satellite drop is between $40 \%$ and $50 \%$ of the equilibrium drop diameter, $D_{0}$; this means that the mass of the satellite drop is about $10 \%$ of the total mass of the drop, therefore the equivalent drop diameter of the drop after the satellite droplet separation is about $96 \%$ of the initial equilibrium diameter.

A second impact morphology peculiar of polymer solution drops can be observed at high Weber numbers. When the drop reaches maximum spreading, satellite droplets are formed around the disk perimeter due to the rim instability; in Newtonian drops, this instability eventually evolves into drop splashing. In the case of polymer solutions, a liquid bridge prevents the separation of the satellite droplets from the lamella, as shown in Figure 3; although the stretching of the liquid bridge is less than in the case of the single satellite drop filament, its elasticity is sufficient to recall the satellite droplets and prevent splashing or breakup. This impact morphology is not observed in Newtonian drops, and can be labelled as partial splashing (or semi-splashing). 


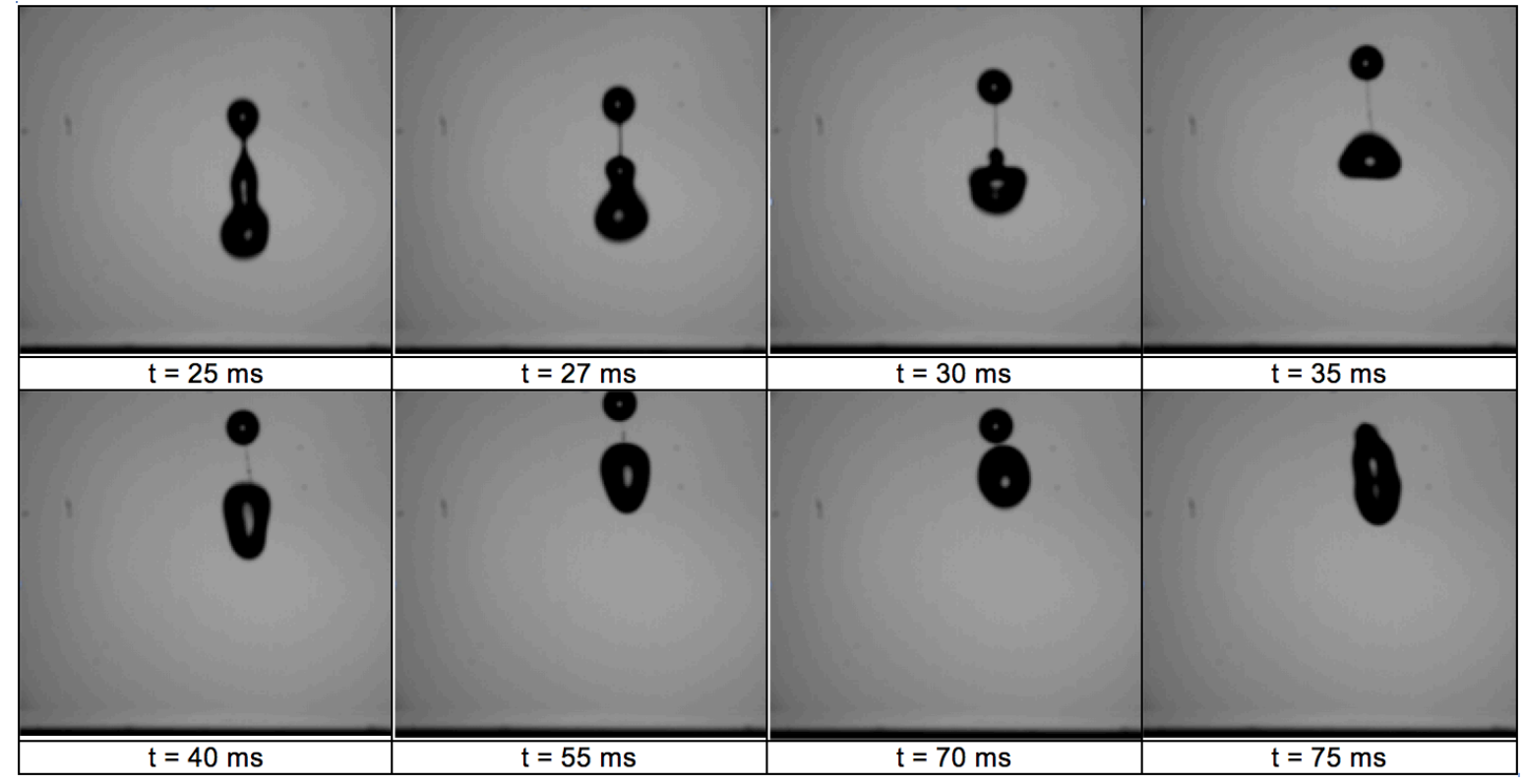

Figure 2. Single satellite drop morphology observed during the impact of a PEO solution drop (concentration: 100ppm; molecular weight: $4 \mathrm{MDa}$ ) impacting on a surface at $\mathrm{T}=400^{\circ} \mathrm{C}$ with $\mathrm{We}=80$; the time origin is the moment of impact.

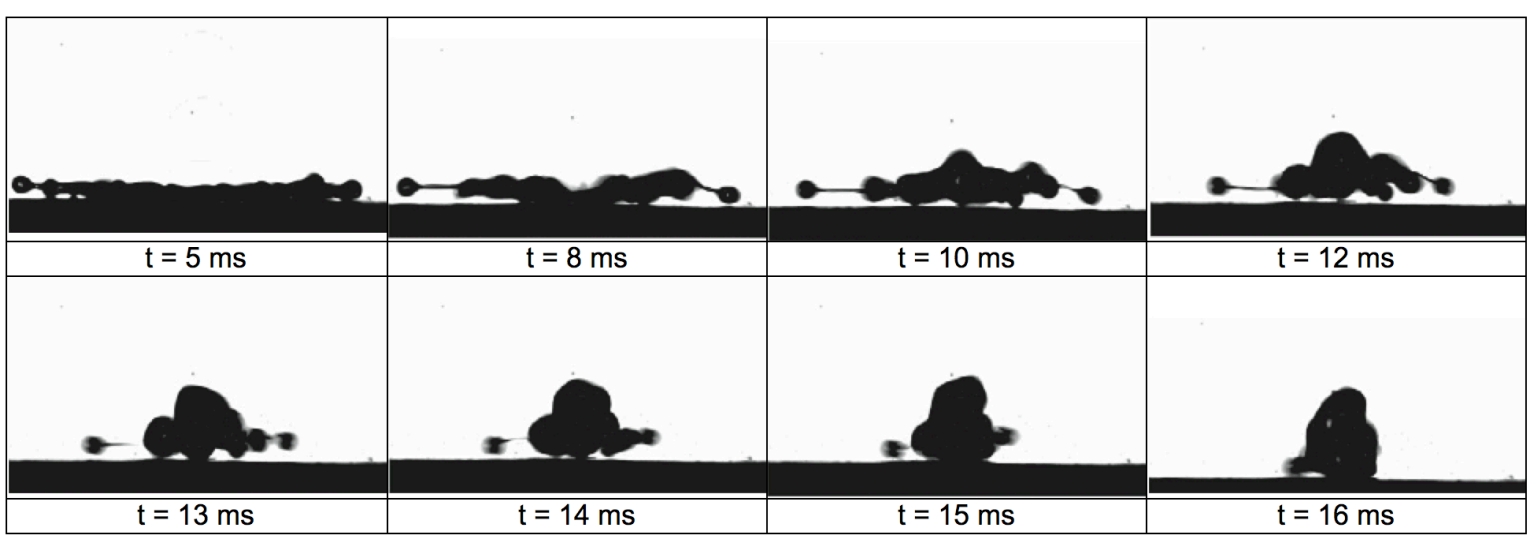

Figure 3. Semi-splash morphology observed during the impact of a PEO solution drop (concentration: 300ppm; molecular weight: $4 \mathrm{MDa}$ ) impacting on a surface at $\mathrm{T}=400^{\circ} \mathrm{C}$ with $\mathrm{We}=200$; the time origin is the moment of impact.

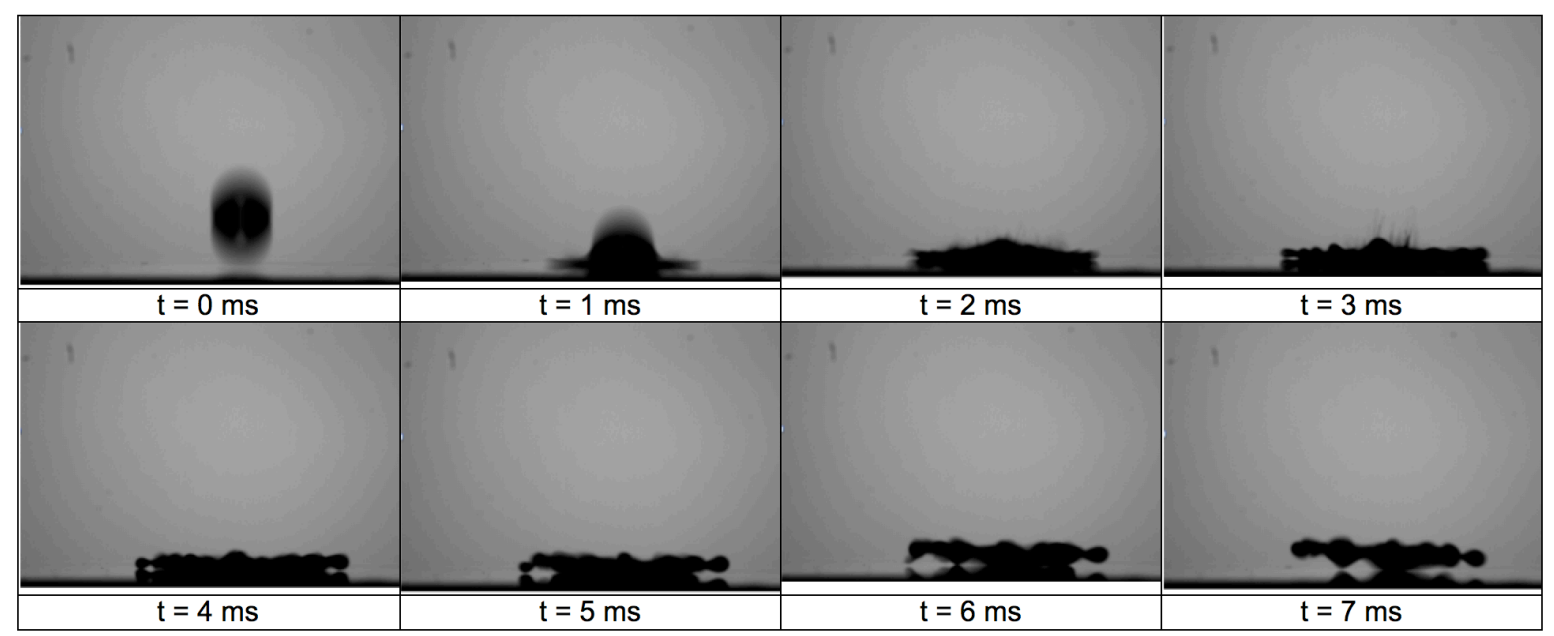

Figure 4. Semi-spray morphology observed during the impact of a PEO solution drop (concentration: 200ppm; molecular weight: $4 \mathrm{MDa}$ ) impacting on a surface at $\mathrm{T}=250^{\circ} \mathrm{C}$ with $\mathrm{We}=100$; the time origin is the moment of impact. 


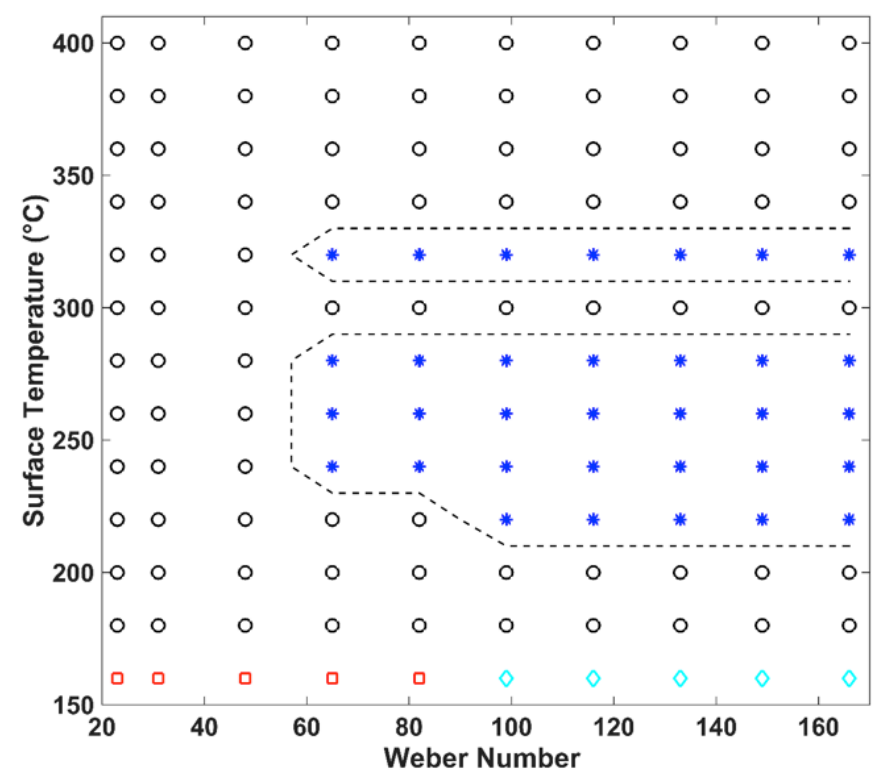

Figure 5. Impact regime map obtained for a concentration of $200 \mathrm{ppm}$ and a molecular weight of $4 \mathrm{MDa}$; regimes shown: SA $(\square), \operatorname{RSA}(\diamond), \mathrm{R}(0)$, and $\mathrm{R}^{*}(*)$.

The formation of liquid bridges preventing the separation of satellite droplets also affects the secondary atomization regime, as shown in Figure 4. In this case, satellite droplets are sprayed out of the spreading drop free surface due to the bursting of vapour bubbles produced at nucleation sites on the impact surface; however, shortly after their ejection all satellite droplets forming the spray are pulled back into the main drop, hence this morphology can be labelled "semi-spray". The phenomenon has an overall duration of a few milliseconds therefore it is very difficult to detect and analyse.

Figure 5 shows the impact regime map relative to a dilute PEO solution with concentration of 200 ppm and molecular weight of the PEO of $4 \mathrm{MDa}$. In the range of parameters considered, the impact regimes observed are secondary atomization (SA), rebound with secondary atomisation (RSA), dry rebound (R), and semi-spray $\left(R^{\star}\right)$. Thus, the map is significantly different with respect to the map obtained for drops of pure water [13]; the dominant impact morphology, observed for most combinations of surface temperature and Weber number, is dry rebound, meaning that the polymer additive strongly inhibits both secondary atomization and splashing.

The effect of polymer concentration is clearly seen upon comparing the map in Figure 5 with the impact regime maps for a molecular weight of 4MDa and PEO concentrations of $100 \mathrm{ppm}$ and $400 \mathrm{ppm}$, displayed in Figure 6 . Reducing the polymer concentration increases the number of different impact morphologies, while for the higher polymer concentration dry rebound is observes almost everywhere, with the semi-spray regime confined to a small region. Upon keeping the molecular weight constant at 4MDa and gradually increasing the concentration from 100 to $200 \mathrm{ppm}$, all break-up and splashing is completely overcome; with the exception of small scale secondary atomisation which is prevalent at a surface temperature of $160^{\circ} \mathrm{C}$. However, upon increasing the concentration to $400 \mathrm{ppm}$, no secondary atomisation is observed even at surface temperature of $160^{\circ} \mathrm{C}$. For all surface temperatures considered, rebound is the primary impact outcome.

Similarly, the effect of the molecular weight of the polymer can be seen upon comparing the map in Figure 5 with the impact regime maps for a concentration of $200 \mathrm{ppm}$ and molecular weights of $2 \mathrm{MDa}$ and $8 \mathrm{MDa}$, displayed in Figure 7. Within the $2 \mathrm{MDa}(200 \mathrm{ppm})$ impact regime map, secondary atomisation (SA), rebound with secondary atomisation (RSA), rebound $(R)$, semi-spray $\left(R^{*}\right)$ and drop break-up $(B)$ regimes are observed. Upon gradual increase of molecular weight from $2 \mathrm{MDa}$ to $4 \mathrm{MDa}$, the break up regime is completely suppressed; however some secondary atomisation is still present at a surface temperature of $160^{\circ} \mathrm{C}$. Increasing the molecular weight to $8 \mathrm{MDa}$, all secondary atomisation is completely suppressed.

Thus, from a qualitative standpoint, the effect of molecular weight is similar to that of the polymer concentration; low molecular weights enable the development of different impact morphologies, while increasing the molecular weight progressively suppresses secondary atomization and breakup/splashing, until only the dry rebound regime can be observed. 

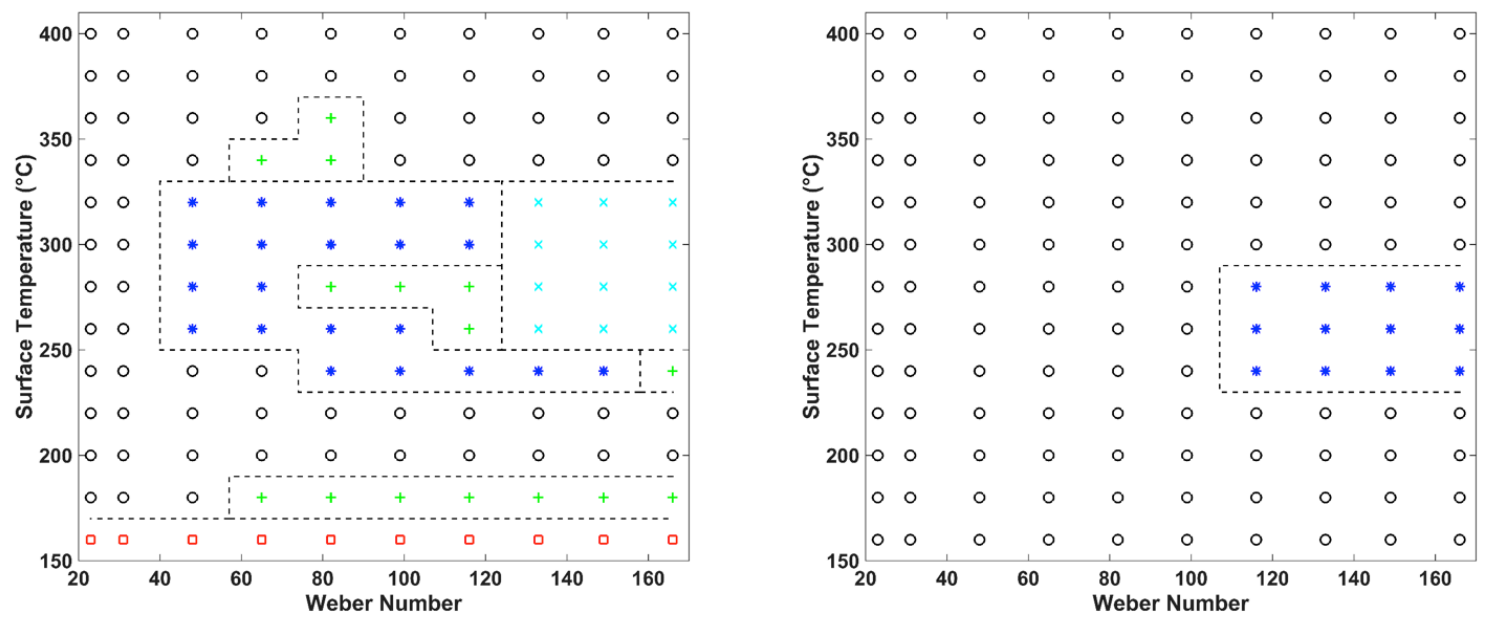

Figure 6. Impact regime maps obtained for a molecular weight of $4 \mathrm{MDa}$ at concentrations of $100 \mathrm{ppm}$ (left) and $400 \mathrm{ppm}$ (right); regimes shown: SA ( $\square), \mathrm{B}(+), \mathrm{R}(0), \mathrm{R}^{*}\left(^{*}\right)$ and $\mathrm{S}(\mathrm{x})$.

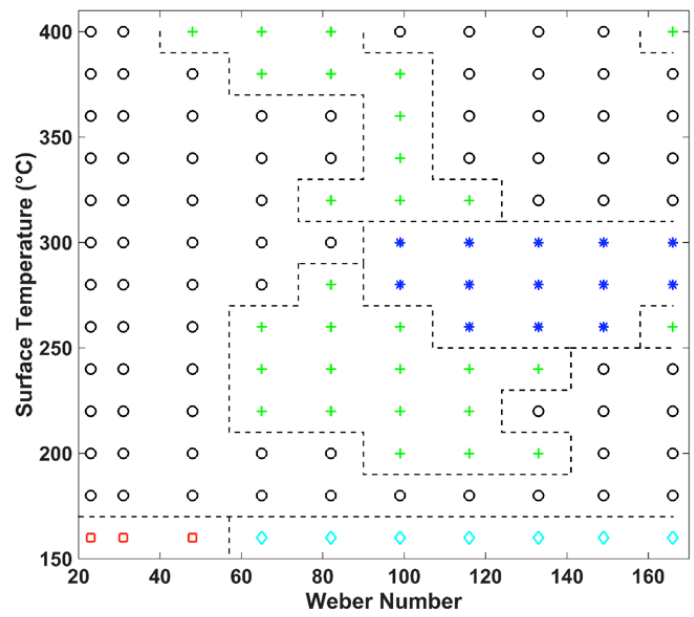

\begin{tabular}{|c|c|c|c|c|c|c|c|c|c|}
\hline \multirow[t]{2}{*}{$40 \mathrm{C}$} & $\begin{array}{lll}0 & 0\end{array}$ & o & 0 & 0 & 0 & 0 & 0 & 0 & o- \\
\hline & $\circ \quad 0$ & 0 & 0 & 0 & 0 & 0 & 0 & 0 & 0 \\
\hline \multirow{2}{*}{350} & 00 & o & 0 & 0 & 0 & 0 & 0 & 0 & 0 \\
\hline & 00 & 0 & 0 & 0 & 0 & 0 & 0 & $\circ$ & 0 \\
\hline \multirow{4}{*}{ 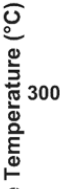 } & 00 & 0 & 0 & 0 & 0 & 0 & 0 & 0 & 0 \\
\hline & $\circ 0$ & $\circ$ & 0 & 0 & $\circ$ & 0 & 0 & 0 & o- \\
\hline & 00 & 0 & $\circ$ & $\circ$ & $\circ$ & $\circ$ & $\circ$ & * & * \\
\hline & $\circ 0$ & $\circ$ & 0 & 0 & 0 & 0 & $\circ$ & * & * \\
\hline \multirow{2}{*}{ 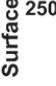 } & 00 & $\circ$ & 0 & 0 & $\circ$ & 0 & $\circ$ & 0 & 0 \\
\hline & 00 & 0 & 0 & 0 & 0 & 0 & 0 & 0 & 0 \\
\hline \multirow[t]{3}{*}{200} & $\circ 0$ & 0 & 0 & 0 & $\circ$ & $\circ$ & 0 & $\circ$ & 0 \\
\hline & $\circ \quad 0$ & 0 & 0 & 0 & 0 & 0 & 0 & 0 & 0 \\
\hline & $\begin{array}{ll}0 & 0\end{array}$ & 0 & 0 & 0 & $\circ$ & 0 & 0 & 0 & 0 \\
\hline 150 & & & & 80 & 10 & 120 & & & \\
\hline
\end{tabular}

Figure 7. Impact regime maps obtained for a concentration of $200 \mathrm{ppm}$ molecular weights of $2 \mathrm{MDa}$ (left) and $8 \mathrm{MDa}$ (right); regimes shown: $S A(\square), B(+), R(0), R^{*}\left({ }^{*}\right)$ and $S(x)$.

The similarity between the effects of the molecular weight and of the polymer concentration on the impact morphology is justified because both of these parameters affect the relaxation time of polymer solutions [21-23]. When the relaxation time is shorter than the characteristic hydrodynamic time scales corresponding to the various impact morphologies, the effect of the polymer additive is negligible, however when the relaxation time and the hydrodynamic time scales are of the same order the the behaviour of polymer solutions becomes significantly different from that of the pure solvent.

\section{Conclusions}

The impact of drops of dilute polyethylene oxide aqueous solutions on a heated polished aluminium surface was investigated by high-speed imaging, for different values of the polymer molecular weight and concentration, with the purpose to evaluate the influence of these parameters on the impact morphology. In addition to impact morphologies observed in Newtonian drops (deposition, rebound, secondary atomisation and breakup/splashing), three new impact regimes have been identified: (i) a single satellite droplet ejected in the direction of bouncing but tethered to the main drop by a thin liquid filament; (ii) a splashing-like behaviour (semi-splashing), where the rim instability generates satellite droplets tethered to the lamella by thin liquid filaments; (iii) a spray-like behaviour (semi-spray), where a fine secondary atomisation generated upon impact is quickly absorbed back into the drop 
globule. Based on experimental observations, five impact regime maps were produced to demonstrate the correlation between varying polymer molecular weight and concentration in suppressing the production of secondary droplets and splashing/breakup.

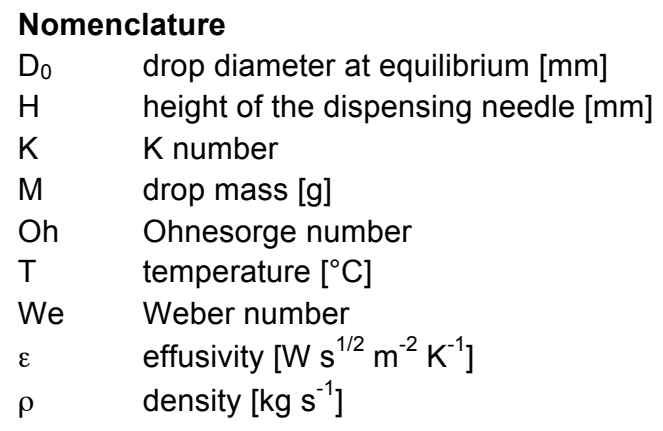

\section{References}

[1] Bai, C., 1995, "Development of methodology for spray impingement simulation".

[2] Bernadin, J.D., Stebbins, C.J. and Mudawar, I., 1997. International Journal of Heat and Mass Transfer, 40(2), pp. 247-267.

[3] Rein, M., 2002, "Interactions between drops and hot surfaces". Springer Vienna.

[4] Lee, S.Y. and Ryu, S.U., 2006. Journal of mechanical science and technology, 20(8), pp. 1101-1117.

[5] Castanet, G., Lienart, T. and Lemoine, F., 2009. International Journal of Heat and Mass Transfer, 52(3), pp. 670-679.

[6] Mundo, C, Sommerfield, M, Tropea, C, 1995. Int. J. Multiphase Flow, 21 (2) 151-173

[7] C. Amiel, 2002, "Application de techniques optiques à l'étude du comportement dynamique et thermique de gouttes en interaction avec une paroi chauffée" Ph.D. Thesis, École Nationale Supérieure de l'Aéronautique et de l'Espace.

[8] Cossali, G.E., Coghe, A., and Marengo, M., 1997. Experiments in Fluids, 22, pp. 463-472.

[9] J.D. Bernardin, I. Mudawar, 1999. The Leidenfrost point: experimental study and assessment of existing models, J. Heat Transfer (Trans. ASME) 121, pp. 894-903.

[10] J.D. Bernardin, I. Mudawar, 1999. A Leidenfrost point model for impinging droplets and sprays, J. Heat Transfer (Trans. ASME) 126, pp. 272-278.

[11] Wang, A.B., Lin, C.H. and Chen, C.C., 2000. Physics of Fluids, 12(6), pp.1622-1625.

[12] Moreira, A.L.N., Moita, A.S. and Panao, M.R., 2010. Progress in energy and combustion science,36(5), pp.554-580.

[13] V. Bertola, 2015, An impact regime map for water drops impacting on heated surfaces, International Journal of Heat and Mass Transfer, vol. 85, pp. 430-437.

[14] Tran, T., Staat, H.J.J., Prosperetti, A., Sun, C. and Lohse, D., 2012. Physical Review Letters, 108(3), 036101

[15] V. Bertola, 2004. Drop impact on a hot surface: effect of a polymer additive, Experiments in Fluids, vol. 37(5), pp. 653-664.

[16] V. Bertola, K. Sefiane, 2005. Controlling secondary atomization during drop impact on hot surfaces by polymer additives, Physics of Fluids, vol. 17(10), 108104.

[17] V. Bertola, 2009. An experimental study of bouncing Leidenfrost drops: comparison between Newtonian and viscoelastic liquids, International Journal of Heat and Mass Transfer, vol. 52, pp. 1786-1793.

[18] V. Bertola, 2014. Effect of polymer concentration on the dynamics of dilute polymer solution drops impacting on heated surfaces in the Leidenfrost regime, Experimental Thermal and Fluid Science, vol. 52, pp. 259-269.

[19] S. Chen, V. Bertola, 2016. Jumps, somersaults and symmetry breaking in Leidenfrost drops, Physical Review E, vol. 94, 021102(R).

[20] S. Chen, V. Bertola, 2016. The impact of viscoplastic drops on a heated surface in the Leidenfrost regime, Soft Matter, vol. 12, pp. 7624-7631.

[21] F.E. Beiley, J.V. Koleske, 1976. Poly(ethylene oxide), New York: Academic Press.

[22] N. P. Cheremisinoff, 1997. Handbook of engineering polymeric materials, New York: Marcel Dekker.

[23] V. N. Kalashnikov, A. N. Askarov, 1989. Relaxation time of elastic stresses in liquids with small additions of soluble polymers of high molecular weights, J Eng Phys Thermophys 57, pp. 874-878. 